\title{
Urgent-start peritoneal dialysis for end-stage renal disease patients: literature review and worldwide evidence-based practice
}

\author{
Atthaphong Phongphithakchai', Phongsak Dandecha', Sukit Raksasuk² and Thatsaphan Srithongkul ${ }^{*}$ (D)
}

\begin{abstract}
The prevalence of end-stage renal disease (ESRD) is on the rise worldwide. Meanwhile, the number of older people requiring dialysis therapy is increasing as a result of this population. We found that starting dialysis in an unplanned manner is a common occurrence, even for patients with nephrology follow-up. Most centers choose hemodialysis with a high rate of central venous catheter use at the time of initiation of dialysis. Current data has found that central venous catheter use is independently associated with increased mortality and high bacteremia rates. Peritoneal dialysis is one option to avoid bacteremia. The International Society for Peritoneal Dialysis guidelines suggests a break-in period of at least two weeks prior to an elective start of peritoneal dialysis, without mentioning urgent-start peritoneal dialysis. For unplanned ESRD patients, it is unrealistic to wait for two weeks before initiating peritoneal dialysis therapy. Urgent-start peritoneal dialysis has been suggested to be a practical approach of prompt initiation of peritoneal dialysis after catheter insertion, which may avoid an increased risk of central venous catheter-related complications, including bacteremia, central venous stenosis, and thrombosis associated with the temporary use of hemodialysis. Peritoneal dialysis is the alternative option, and many studies have presented an interest in urgent-start peritoneal dialysis. Some reports have compared urgent-start hemodialysis to peritoneal dialysis and found that urgent-start peritoneal dialysis is a safe and effective alternative to hemodialysis for an unplanned dialysis patient. This review aims to compare each literature report regarding techniques, prescriptions, outcomes, complications, and costs of urgentstart peritoneal dialysis.
\end{abstract}

Keywords: End-stage renal disease, Peritoneal dialysis, Urgent-start

\section{Background}

To date, the worldwide prevalence of end-stage renal disease (ESRD) is on the rise. According to the United States Renal Data System (USRDS) 2019 annual data report, the prevalence of ESRD continues to rise and reached 746,557 cases in 2017 (vs. 727,912 cases in 2016), representing a 2.6\% increase since 2016 [1]. Unplanned dialysis is the primary concern for many patients who progress

\footnotetext{
*Correspondence: thatsaphan.sri@mahidol.ac.th

${ }^{2}$ Division of Nephrology, Department of Medicine, Faculty of Medicine

Siriraj Hospital, Mahidol University, 2 Wang Lang Road, Bangkok Noi, Bangkok 10700, Thailand

Full list of author information is available at the end of the article
}

to ESRD despite efforts to increase early referral and close monitoring of these patients. Since it cannot be completely prevented, it may contribute to urgent-start dialysis.

Starting urgent dialysis is the urgent initiation of dialysis for unplanned ESRD patients without a pre-established functional vascular access or Tenckhoff (TK) catheter placement. The unplanned start of dialysis is often accomplished by hemodialysis (HD) using a central venous catheter $(\mathrm{CVC})$ that increases the patient's risks of infection and other catheter-related complications [2]. CVC usage is considered suboptimal because CVCs are independently associated with increased mortality and

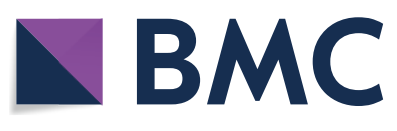

(c) The Author(s) 2021. Open Access This article is licensed under a Creative Commons Attribution 4.0 International License, which permits use, sharing, adaptation, distribution and reproduction in any medium or format, as long as you give appropriate credit to the original author(s) and the source, provide a link to the Creative Commons licence, and indicate if changes were made. The images or other third party material in this article are included in the article's Creative Commons licence, unless indicated otherwise in a credit line to the material. If material is not included in the article's Creative Commons licence and your intended use is not permitted by statutory regulation or exceeds the permitted use, you will need to obtain permission directly from the copyright holder. To view a copy of this licence, visit http://creativecommons.org/licenses/by/4.0/. The Creative Commons Public Domain Dedication waiver (http://creativeco mmons.org/publicdomain/zero/1.0/) applies to the data made available in this article, unless otherwise stated in a credit line to the data. 
high rates of bacteraemia [3, 4]. Unplanned dialysis starts with PD to improve patient outcomes by avoiding CVC usage, of recent increased interest and a possible choice for some patients.

The definition of urgent-start PD (USPD) is using a TK catheter within 14 days after insertion [5]. USPD is a strategy in which patients with advanced chronic kidney disease (CKD) who urgently and unexpectedly require dialysis due to uremia or fluid overload are treated with PD rather than HD, which has been the standard approach worldwide, excluding the use of PD in acute kidney injury (AKI) [5, 6]. Currently, starting renal replacement therapy (RRT) in PD using the TK catheter within $72 \mathrm{~h}$ after the implant with no pre-established HD therapy has been raised [7, 8]. Multiple studies have described the definition of urgent PD as similar but different in the protocols and prescriptions of USPD that have been reported in the literature. This review article aims to clarify the current literature of USPD regarding the catheter insertion approach techniques, protocols, prescriptions, complications, and outcomes in order to suggest a better way to apply it in real-life situations.

\section{The catheter approach technique and types of catheters}

One of the crucial steps to achieve successful urgent-start PD is catheter insertion. Many studies described the outcomes of various catheter insertion techniques, including percutaneous surgery (Seldinger technique), open surgery, and Laparoscopic surgery. Silva et al. evaluated technical survival in 154 Brazilian patients who started early (40 patients) and planned PD (114 patients). The Early-start PD group was defined as a break-in period from 3 to 14 days. All patients underwent TK catheter insertion by nephrologists (Seldinger technique) or video-laparoscopically by surgeons. The type of catheter was a straight, double-cuffed TK catheter. There is no difference in technical survival between both groups at 381 days of follow-up period [9]. In China, the largest USPD cohort was performed by $\mathrm{Xu}$ et al. Straight double-cuff TK catheters were used in the study. Major of the patients underwent open surgery for TK placement by a nephrologist. However, Laparoscopic was chosen for patients who underwent simultaneous hernia repair or had a history of multiple abdominal surgeries. No immediate post-operative complications were observed [10]. Similarly, a large 10-year retrospective study in 2,059 ESRD patients receiving USPD (within 14 days) also showed a favorable outcome. Double-cuff TK catheters were used in this study. Nephrologists did open laparotomy in all patients. A double purse-string suture technique on the posterior rectus sheath and parietal peritoneum was performed to prevent leakage. Only $0.1 \%$ of hemorrhage complication was found [11]. Therefore, results emphasized that the percutaneous and open surgery technique by the experienced team may reduce the complication.

Form literature reviews demonstrated that laparoscopic insertion allowed early PD initiation with good outcomes. Toda and colleagues retrospectively analyzed 54 ESRD patients in Japan who underwent a laparoscopic TK insertion. The patients were divided into two groups according to PD started (early; within seven days, and late; after seven days). The type of catheter was a doublecuffed swan neck TK catheter. A purse-suture was placed on the internal cuff, and the rectus muscle was closed. Results showed that neither group experienced increased infection-related complications nor PD withdrawal [12].

The study from Ghaffari et al. demonstrated the outcomes of USPD performed by radiology interventionists. Dual-cuff, swan-neck, coiled TK catheters were inserted by percutaneous placement. In the USPD group, the hematoma was found in 1 of 18 patients (5.6\%) [13]. Moreover, this study showed the well-structured patient selection process for choosing the appropriate USPD program candidate (Fig. 1).

Htay et al. conducted a systematic review from 3144 participants, 42 studies to evaluate the techniques of TK catheter implantation and catheter types in lowering the risk of PD-related peritonitis. The systematic review included 18 studies comparing different catheter insertion techniques, 22 studies comparing catheter types, one study of immobilization techniques, and one study for different break-in periods. The results showed that catheter insertion by laparoscopy compared to laparotomy probably makes little or no difference in the risks of peritonitis (relative risk [RR] 0.90; 95\% confidence interval [CI] 0.59-1.35), catheter removal/replacement (RR 1.20; 95\% CI 0.77-1.86), technique failure (RR 0.71; 95\% CI $0.47-1.08$ ), and death (RR 1.26; 95\% CI 0.72-2.20). Moreover, no TK catheter design showed a superior outcome to others [14].

The outcome of USPD among different catheter insertion techniques was summarized in Table 1. All studies in Table 1 included PD initiation within seven days after TK catheter placement and clearly defined the catheter insertion technique. The outcome had infectious, non-infectious complications, patient and catheter survival in USPD groups. Although there are no current studies directly comparing results of different insertion methods in USPD, From the evidence demonstrated the low incidences of post-operative complications in USPD. Hence, the catheter implantation for USPD conducted by trained physicians was probably safe, and the PD catheter approach technique is not an obstacle to USPD. However, USPD may increase the risk 


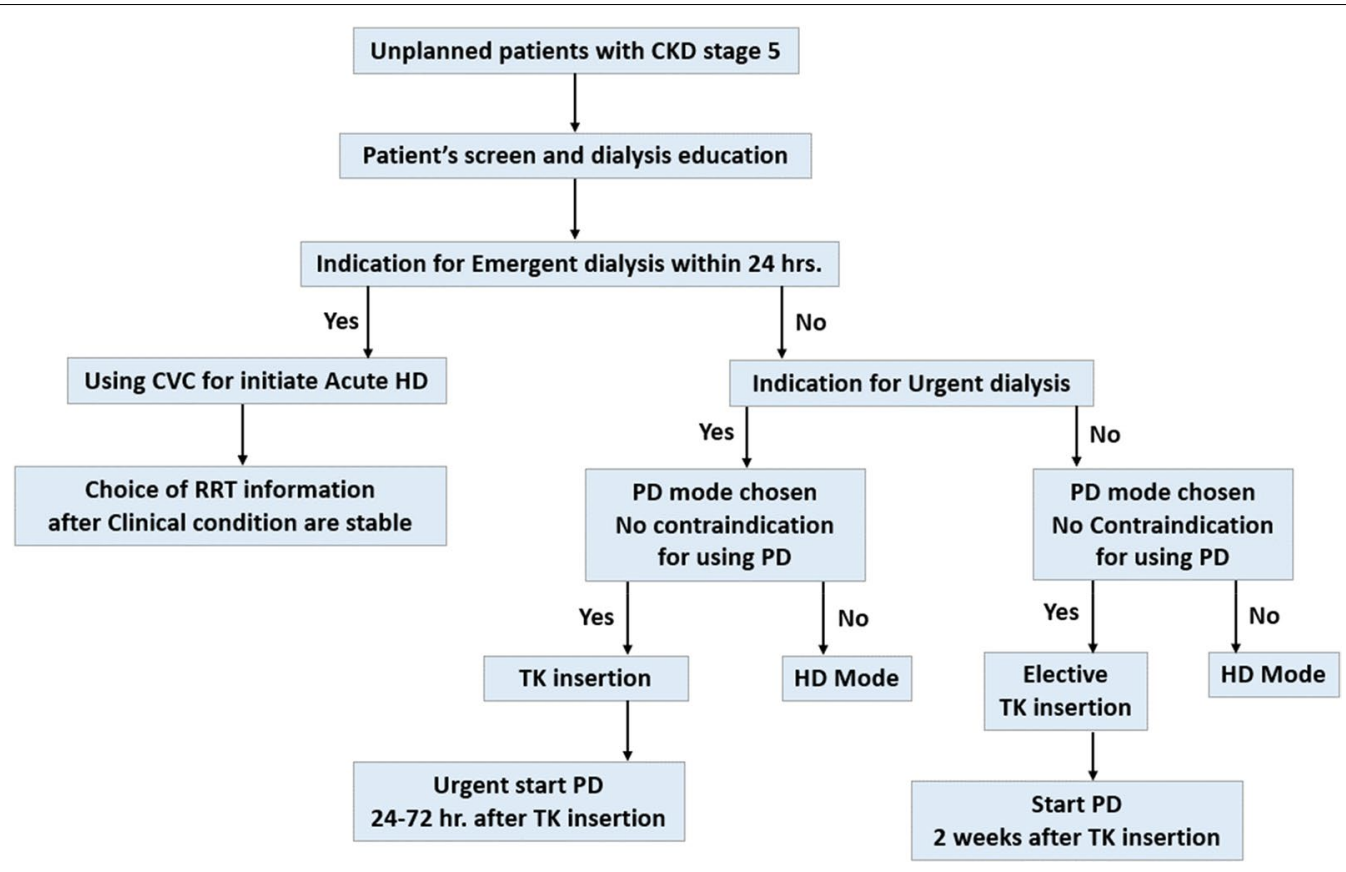

Fig. 1 The algorithm of dialysis initiation in unplanned CKD stage 5 patients

of post-operative complications, such as leakage and mechanical complication.

\section{Program and prescription}

The PD program and prescription had been emphasized as the second step after catheter insertion of successful USPD. Alkatheeri et al. described the Canadian renal program, one of the most successful USPD protocols, in which PD was initiated within two weeks of catheter insertion. Initially, the dwell volume was $1000-1200 \mathrm{~mL}$ in all patients, gradually increasing to $1900 \mathrm{~mL}$ by the end of three to four weeks if there were no complications. A supine position was enforced, using a cycler for the initial two weeks and no day dwelling for four to six weeks. No one in this study had to switch modalities [15]. Wang et al. conducted a study that compared the efficiency and complications between intermittent peritoneal dialysis (IPD) and automatic peritoneal dialysis (APD) with tidal volume for USPD. The IPD protocol consisted of $0.5 \mathrm{~L}$ of the intraperitoneal volume and $30 \mathrm{~min}$ of dwell time per exchange on the first and second days, and the prescription was increased to $800 \mathrm{~mL}$ for $1 \mathrm{~h}$ on the third and the fourth days, $1000 \mathrm{~mL}$ for $2 \mathrm{~h}$ on the fifth and sixth days, and then $2000 \mathrm{~mL}$ for four hours on the seventh day. The total dialysis volume was $4 \mathrm{~L}$ every day. The tidal-APD protocol entailed $9 \mathrm{~h}$ of treatment in the supine position with $75 \%$ of tidal volume. The cyclers deliver eight cycles of $800 \mathrm{~mL}$ fill volume with $68 \mathrm{~min}$ of dwell time. Total dialysate volume is $5 \mathrm{~L}$ daily. Both groups converted to full PD prescriptions after one week. The result was no significant difference in technique survival between the groups [16]. Ranganathan et al. evaluated 122 patients with different times of PD initiation, which were within one week, two weeks, and four weeks after TK insertion. All patients received the same prescription but varied PD intervals. On the first day, PD was initiated at a low intraperitoneal volume $(1 \mathrm{~L})$, one-hour dwell, four exchanges per day, and increased to $500 \mathrm{~mL}$ and 30 min dwell time on second and third days. On days 4 and 5, PD consisted of $2 \mathrm{~L}$ exchanges every two hours. Home CAPD was started on day 6. The technique failure was found significantly high in patients who started PD at four weeks (20\%) compared with other groups, which may be explained by a higher number of diabetes patients in this group [17]. Nayak et al. reported the outcome of USPD that started within $48 \mathrm{~h}$ after presentation. The patients were prescribed APD; fill volumes per cycle ranged between $500-750 \mathrm{~mL}$ and $30-45 \mathrm{~min}$ dwell times, depending on their individualized needs. Total daily PD volume was 10-15 L, with an entire duration of therapy of 8-10 h. After one week, the treatment can be continued using APD or changed to CAPD. It was found that technique survival was $88.2 \%$ at 90 days [18]. Therefore, there is supporting evidence that USPD starting with low intraperitoneal volume, especially using APD in the supine position, is helpful to prevent leakage complications. 


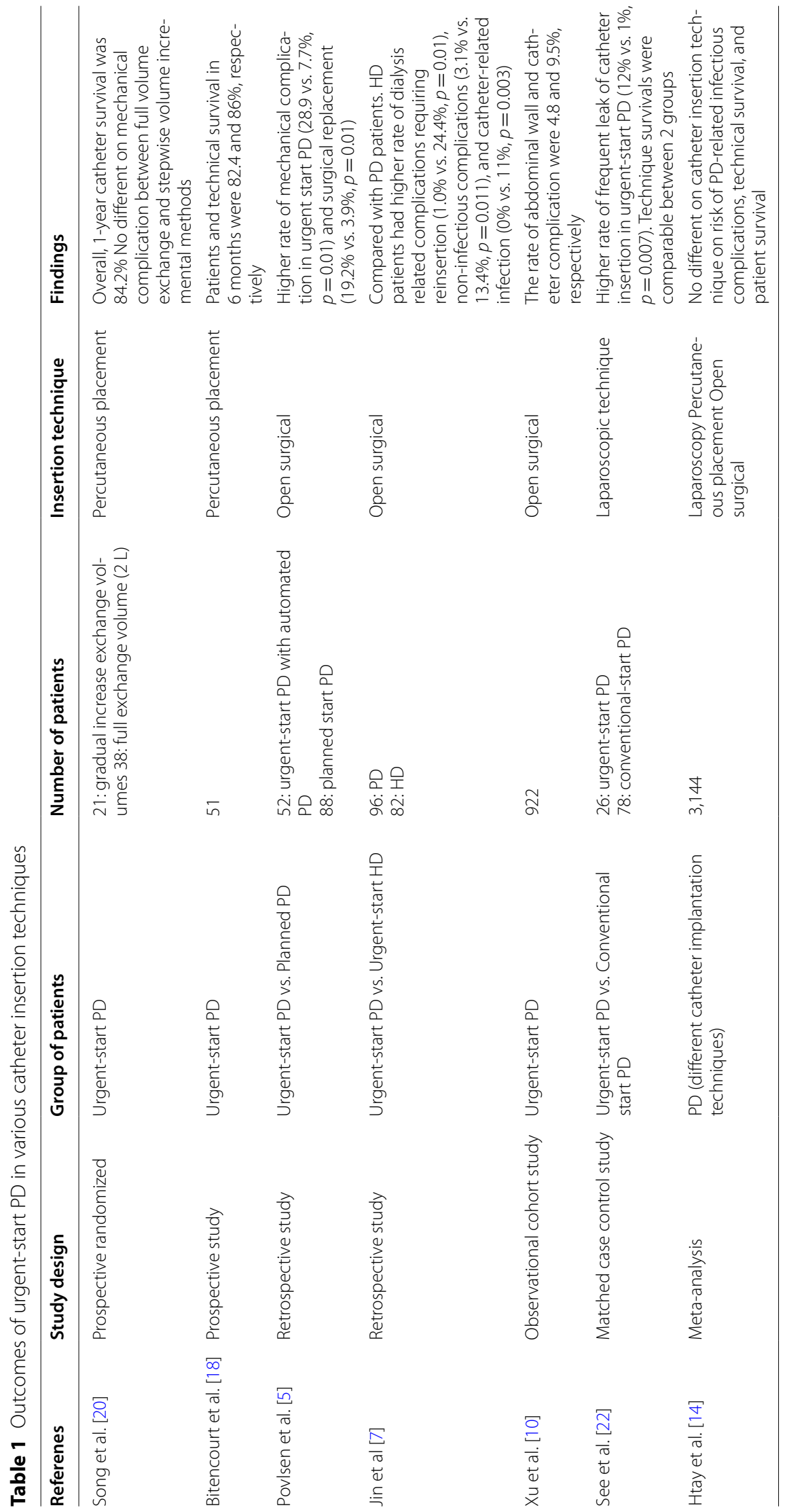


Moreover, initial high volume prescription has also been used in USPD. Dias et al. conducted high-volume PD (HVPD) within $72 \mathrm{~h}$ after catheter insertion. HVPD in this study was defined by $24 \mathrm{~h}$ per session, a prescribed dialysis dose (Kt/Vurea) of 0.5 , and a large volume of dialysate $(30 \mathrm{~mL} / \mathrm{kg})$. All patients received HVPD for three consecutive days for achieving metabolic control before continuing PD prescription adjusted by the patient's condition. Technique and patient survival rates were $86 \%$ and $82.4 \%$ in the first six months, respectively [19]. The results suggest that HVPD did not increase complications and can be used in the unplanned setting.

There is only a randomized comparative study that evaluates the USPD outcome between low-volume and full-volume initiation. In the low volume, filled volume was gradually increased from $500 \mathrm{~mL}$ per three hours to full volume over 13 days. In contrast, the full-volume exchange per six hours was performed immediately after catheter insertion in the full volume group. The occurrence of catheter-related complications was no different between groups. The actual one-year catheter survival was comparable $(85.7 \%$ and $84.2 \%$ in group 1 and group 2 , respectively) [20]. This study showed that initial fullvolume exchange increased neither short nor long-term complications.
The studies mentioned above show that the program and prescription of USPD are necessary to achieve success. USPD begins with a low fill volume of $500-750 \mathrm{ml}$, $1.5-3 \mathrm{~h}$ of dwell time (4-8 cycles per day) on the first day in the supine position. Then, the volume is gradually increased to $250-500 \mathrm{ml}$ in the following 3-5 days until the maximum tolerable volume is reached within 10 days may be appropriate to prescribe. The solution's tonicity should be determined by the patient's volume status. The algorithm for the initial prescription of USPD is shown in Fig. 2 [15-19]. Overall, prescriptions mostly consist of IPD with low fill volumes in the supine posture, depending on the patient's condition; however, high-volume PD or standard fill volume can be performed under closed monitoring. Moreover, the prescription could be adjusted to give more flexibility, and proper therapy monitoring by a well-trained PD team is essential.

\section{Outcomes and complications}

In the last decade, several studies evaluating outcomes and complications of USPD have been reported. Zang and colleagues studied the outcome in elderly patients who were initiated USPD or urgent-start hemodialysis (USHD). USPD was associated with fewer complications and better survival than USHD in the elderly. Dialysisrelated complications within 30 days were significantly

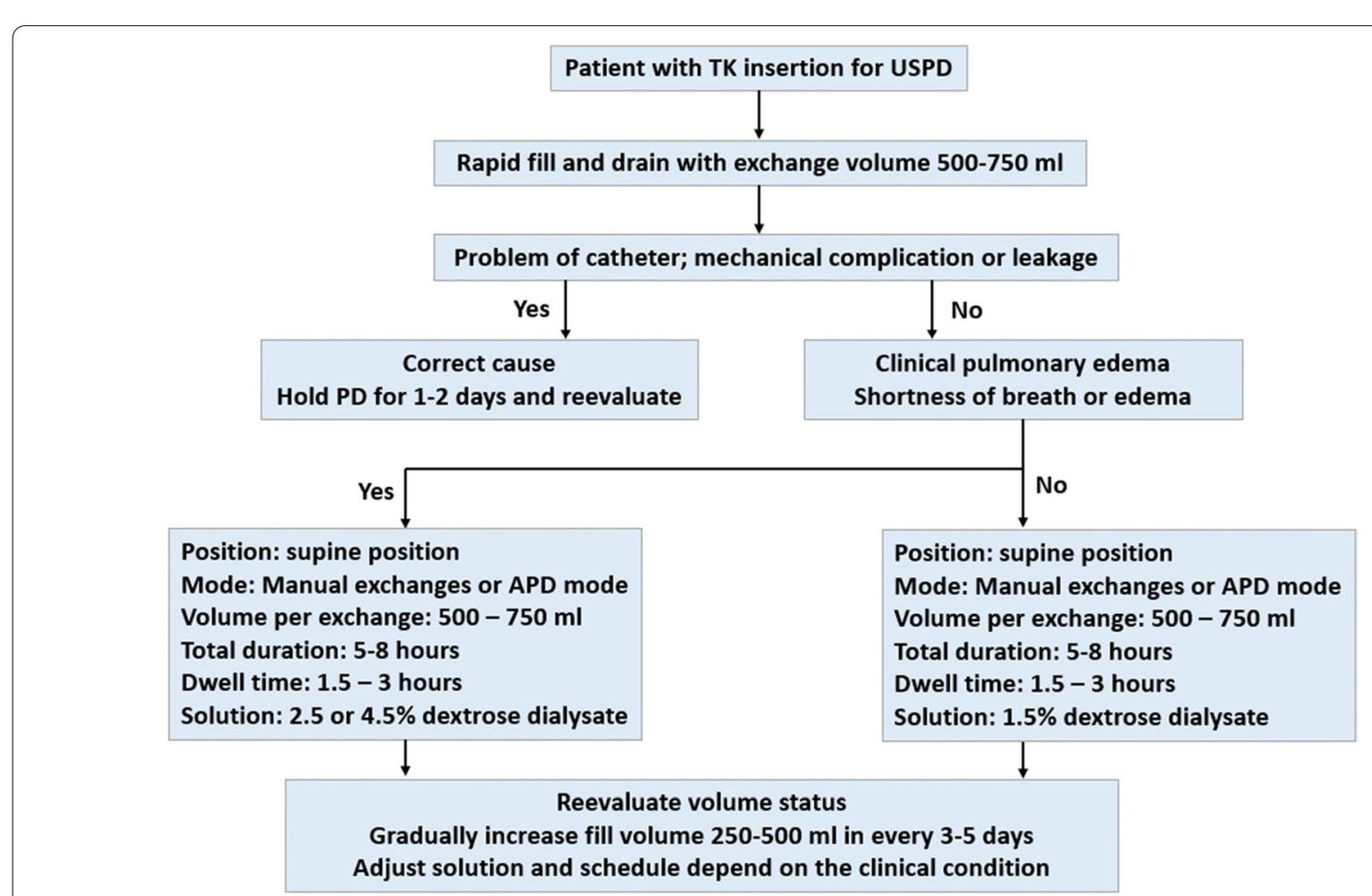

Fig. 2 The algorithm of initial PD prescription in Urgent-start PD 
lower in the USPD group than the USHD group (4.5\% vs. $10.7 \%, p=0.031$ ). The 1 and 3 -year survival rates were $91.4 \%$ and $64.8 \%$ in the USPD group and $85.7 \%$ and $57.8 \%$ in the USHD group, respectively $(p=0.023)$. Multivariable Cox regression analysis showed that USHD was independently associated with death (hazard ratio $[\mathrm{HR}]=2.22, p=0.004)[21]$.

Catheter migration and leakage are the most important concern for USPD initiation, especially in elderly patients. See et al. studied Australian ESRD patients who were started USPD with a median time 4 days after catheter insertion. The patients in USPD group were more likely to develop leakage complications $(12 \%$ vs. $1 \%, p=0.047)$ and catheter migration $(12 \%$ vs. $1 \%$, $p=0.047$ ) The technique survival rate for urgent- and conventional-start PD was $68 \%$ and $80 \%$ at 1 year, $48 \%$ and $38 \%$ at 3 years, respectively. There was no difference in the rate of infectious complications between the groups at either time point [22]. Wojtaszek et al. also compared the short- and long-term outcomes between 35 unplanned and 94 planned dialysis patients in Poland. The mean time between catheter implantation and PD start was $3.5 \pm 2.3$ days in the USPD and $16.2 \pm 1.7$ days in the planned PD group. Early mechanical complications were observed more often in the urgent-start group ( 29 vs. $4 \%, p=0.00005$ ). Technical survival was excellent in both groups, $100 \%$ and $96 \%$ at 3 months, $97 \%$ and $87 \%$ at 12 months, and $83 \%$ and $76 \%$ at the end of the observation for USPD and planned dialysis, respectively [23]. These percentages of complications are higher than reported by the other experienced USPD centers, but it did not affect the patient and technique survival. Consequently, It appears that USPD is associated with equivalent rates of early and late infectious complications and marginally higher rates of acceptable mechanical complications.

In general, concerning the management of abdominal wall complications, patients were evaluated regarding whether dialysis therapy should be temporarily suspended. If PD can be continued, adjusting PD regimens with lower infusion volumes in a proper position (supine position for hernia and sitting position for hydrothorax) should be appropriated or transferred to temporarily HD for healing the leakage sites. After the leakage was resolved, PD could be resumed. For the patient who had recurrent leakage, surgical repair should be considered $[10,11]$. In cases of catheter-related mechanical complications when drain failure occurred, X-ray examination and/or ultrasonography were performed to determine catheter position. A conservative treatment strategy (e.g., laxative) was used for primary treatment in catheter migration. If malposition persisted or omental wrapping, surgical intervention of the malfunctioning catheter would be performed. Intraluminal installation of thrombolytic drugs can be used in case of catheter obstruction by fibrin clots. Additionally, complications management should be taken jointly by a nephrologist, PD nurse, surgical team, and radiology interventionist.

\section{Cost}

Liu et al. conducted a study for the economic evaluation of USPD and USHD in the United States over the first 90 days of treatment from a provider perspective. The estimated per-patient cost over the first 90 days for USPD was $\$ 16,398$ ( $15 \%$ for dialysis access, $48 \%$ for dialysis services, $37 \%$ for hospitalization). For USHD, the total perpatient cost was $\$ 19,352$ (27\% for dialysis access, $42 \%$ for dialysis services, and $31 \%$ for initial hospitalization). As a result, USPD provided lower costs to hospitals and clinics during the first 90 days of dialysis than USHD [24]. The primary cost savings result from lower costs associated with peritoneal access establishment. There may also be saved through the differences in costs associated with infectious complications.

\section{Conclusions}

Urgent-start peritoneal dialysis (USPD) provided a comparable outcome with a marginally higher mechanical complication rate than the standard PD initiation. The catheter type and insertion technique did not affect the outcome. To avoid complications, starting with low intraperitoneal volume in the supine position and titrating to full volume is appropriate. However, a full volume or high volume PD may be used depending on the patient's requirement. USPD provided more cost-effectiveness and less infectious complication than urgent start hemodialysis. Therefore, USPD may be considered a primary strategy in unplanned dialysis patients who need the urgent start of dialysis.

\section{Abbreviations \\ USPD: Urgent-start peritoneal dialysis; ESRD: End-stage renal disease; PD: Peritoneal dialysis; CVC: Central venous catheter; HD: Hemodialysis; USHD: Urgent-start hemodialysis.}

\section{Acknowledgements}

Not applicable.

\section{Authors' contributions}

TS was involved in the conceptualization and design of the manuscript. AP wrote the first draft. TS has worked with AP on its developments. TS and PD critically revised the manuscript for important intellectual content. All authors read and approved the final review article.

\section{Funding}

Not applicable. 


\section{Availability of data and materials}

Data sharing not applicable to this article as no data-sets were generated or analyzed during the current study.

\section{Declarations}

\section{Ethics approval and consent to participate} Not applicable.

\section{Consent for publication}

Not applicable.

\section{Competing interests}

The authors declare that they have no competing interests.

\section{Author details}

${ }^{1}$ Division of Nephrology, Department of Internal Medicine, Faculty of Medicine, Prince of Songkla University, Hat Yai, Songkhla, Thailand. ${ }^{2}$ Division of Nephrology, Department of Medicine, Faculty of Medicine Siriraj Hospital, Mahidol University, 2 Wang Lang Road, Bangkok Noi, Bangkok 10700, Thailand.

\section{Received: 1 September 2021 Accepted: 11 November 2021}

Published online: 04 December 2021

\section{References}

1. Saran R, Robinson B, Abbott KC, Bragg-Gresham J, Chen X, Gipson D, et al. US renal data system 2019 annual data report: epidemiology of kidney disease in the United States. Am J Kidney Dis. 2020;75(1 Suppl 1):A6-7. https://doi.org/10.1053/j.ajkd.2019.09.003.

2. Sahli F, Feidjel R, Laalaoui R. Hemodialysis catheter-related infection: rates, risk factors and pathogens. J Infect Public Health. 2017;10(4):403-8. https://doi.org/10.1016/j.jiph.2016.06.008.

3. Perl J, Wald R, McFarlane P, Bargman JM, Vonesh E, Na Y, et al. Hemodialysis vascular access modifies the association between dialysis modality and survival. J Am Soc Nephrol. 2011;22(6):1113-21. https://doi.org/10. 1681/ASN.2010111155.

4. Htay H, Johnson DW, Craig JC, Teixeira-Pinto A, Hawley CM, Cho Y. Urgentstart peritoneal dialysis versus conventional-start peritoneal dialysis for people with chronic kidney disease. Cochrane Database Syst Rev. 2020;12(12):CD012913. https://doi.org/10.1002/14651858.CD012913. pub2.

5. Povlsen JV, Ivarsen P. How to start the late referred ESRD patient urgently on chronic APD. Nephrol Dial Transplant. 2006;21 Suppl 2:ii56-59. https:// doi.org/10.1093/ndt/gfl192.

6. Tunbridge M, Cho Y, Johnson DW. Urgent-start peritoneal dialysis: is it ready for prime time? Curr Opin Nephrol Hypertens. 2019;28(6):631-40. https://doi.org/10.1097/MNH.0000000000000545.

7. Jin H, Fang W, Zhu M, Yu Z, Fang Y, Yan H, et al. Urgent-start peritoneal dialysis and hemodialysis in ESRD patients: complications and outcomes. PLOS ONE. 2016;11(11): e0166181. https://doi.org/10.1371/journal.pone. 0166181.

8. Jin H, Ni Z, Che X, Gu L, Zhu M, Yuan J, et al. Peritoneal dialysis as an option for unplanned dialysis initiation in patients with end-stage renal disease and diabetes mellitus. Blood Purif. 2019;47(1-3):52-7. https://doi. org/10.1159/000493176.

9. Silva BC, Adelina E, Pereira BJ, Cordeiro L, Rodrigues CE, Duarte RJ, et al. Early start peritoneal dialysis: technique survival in long-term follow-up. Kidney Blood Press Res. 2018;43(6):1699-705. https://doi.org/10.1159/ 000495386.

10. Xu D, Liu T, Dong J. Urgent-start peritoneal dialysis complications: prevalence and risk factors. Am J Kidney Dis. 2017;70(1):102-10. https://doi. org/10.1053/j.ajkd.2016.12.021.

11. Ye H, Yang X, Yi C, Guo Q, Li Y, Yang Q, et al. Urgent-start peritoneal dialysis for patients with end stage renal disease: a 10-year retrospective study. BMC Nephrol. 2019;20(1):238. https://doi.org/10.1186/s12882-019-1408-9.

12. Toda N, Yanagita M, Yokoi H. Urgent-start peritoneal dialysis after laparoscopic dialysis catheter implantation: a single-center experience. Ren Replace Ther. 2019;5:43. https://doi.org/10.1186/s41100-019-0239-2.
13. Ghaffari A. Urgent-start peritoneal dialysis: a quality improvement report. Am J Kidney Dis. 2012;59(3):400-8. https://doi.org/10.1053/j.ajkd.2011.08. 034.

14. Htay H, Johnson DW, Craig JC, Schena F, Strippoli GFM, Tong A, et al. Catheter type, placement and insertion techniques for preventing catheter-related infections in chronic peritoneal dialysis patients. Cochrane Database Syst Rev. 2019;5:CD004680. https://doi.org/10.1002/14651858. CD004680.pub3.

15. Alkatheeri AMA, Blake PG, Gray D, Jain AK. Success of urgent-start peritoneal dialysis in a large Canadian renal program. Perit Dial Int. 2016;36(2):171-6. https://doi.org/10.3747/pdi.2014.00148.

16. Wang C, Fu X, Yang Y, Deng J, Zhang HQ, Deng HM, et al. A Comparison between intermittent peritoneal dialysis and automatic peritoneal dialysis on urgent peritoneal dialysis. Am J Nephrol. 2017;45(6):540-8. https:// doi.org/10.1159/000477178.

17. Ranganathan D, John GT, Yeoh E, Williams N, O'Loughlin B, Han T, et al. A randomized controlled trial to determine the appropriate time to initiate peritoneal dialysis after insertion of catheter (timely PD study). Perit Dial Int. 2017;37(4):420-8. https://doi.org/10.3747/pdi.2016.00066.

18. Nayak K, Subhramanyam S, Pavankumar N, Antony S, Khan MA. Emergent start peritoneal dialysis for end-stage renal disease: outcomes and advantages. Blood Purif. 2018;45:313-9. https://doi.org/10.1159/000486543.

19. Bitencourt Dias D, Mendes ML, Burgugi Banin V, Barretti P, Ponce D. Urgent-start peritoneal dialysis: the first year of brazilian experience. Blood Purif. 2017;44(4):283-7. https://doi.org/10.1159/000478970.

20. Song JH, Kim GA, Lee SW, Kim MJ. Clinical outcomes of immediate fullvolume exchange one year after peritoneal catheter implantation for CAPD. Perit Dial Int. 2000;20(2):194-9.

21. Zang X, Du X, Li L, Mei C. Complications and outcomes of urgent-start peritoneal dialysis in elderly patients with end-stage renal disease in China: a retrospective cohort study. BMJ Open. 2020;10(3): e032849. https://doi.org/10.1136/bmjopen-2019-032849.

22. See EJ, Cho Y, Hawley CM, Jaffrey LR, Johnson DW. Early and late patient outcomes in urgent-start peritoneal dialysis. Perit Dial Int. 2017;37(4):4149. https://doi.org/10.3747/pdi.2016.00158.

23. Wojtaszek E, Grzejszczak A, Grygiel K, Małyszko J, Matuszkiewicz-Rowińska J. Urgent-start peritoneal dialysis as a bridge to definitive chronic renal replacement therapy: short- and long-term outcomes. Front Physiol. 2019;9:1830. https://doi.org/10.3389/fphys.2018.01830.

24. Liu FX, Ghaffari A, Dhatt H, Kumar V, Balsera C, Wallace E, et al. Economic evaluation of urgent-start peritoneal dialysis versus urgent-start hemodialysis in the United States. Medicine (Baltimore). 2014;93(28): e293. https://doi.org/10.1097/MD.0000000000000293.

\section{Publisher's Note}

Springer Nature remains neutral with regard to jurisdictional claims in published maps and institutional affiliations.

Ready to submit your research? Choose BMC and benefit from:

- fast, convenient online submission

- thorough peer review by experienced researchers in your field

- rapid publication on acceptance

- support for research data, including large and complex data types

- gold Open Access which fosters wider collaboration and increased citations

- maximum visibility for your research: over $100 \mathrm{M}$ website views per year

At BMC, research is always in progress.

Learn more biomedcentral.com/submissions 